\title{
ETH Zürich und die Basler Chemie - eine grosse Tradition
}

\author{
Erweiterte und ergänzte Fassung eines Vortrages beim Symposium \\ "Partnerschaft in der Forschung" der ETH Zürich in Basel, \\ Freitag, 11. März 2005
}

\author{
Dieter Seebach*
}

\section{ETH Zurich and the Chemical Industry in Basel - A Great Tradition}

\begin{abstract}
A historic account of the traditional relationship between the Chemistry Department of ETH Zürich and the Chemical Industry in Basel is presented. The development of ETH Zürich from a Swiss polytechnical school to one of the leading research institutions in the world and the growth of the Swiss companies in the 'Dreiländereck' from dye-stuff producers to global top-players in the pharma- and agro businesses are shown to be closely connected. The strong personality of chemists such as Ruzicka, Reichstein, Karrer, Stoll, and Prelog is correlated with these unique parallel evolutions. Recent changes, both in chemical research and in chemical industry, have led to a new situation in which the ETH Zürich chemistry department competes globally for the best students and researchers, and in which the companies do not only business, but also research, development, production, and thus hiring, on a global scale. As chemistry moves into the bio-, medical-, and material sciences we have to safeguard a strong emphasis in teaching the basics of our science (inorganic, organic, physical chemistry, analysis and synthesis - the core of chemistry), and at the same time make sure that our students and researchers learn the language(s) of the 'neighboring' sciences to be optimally prepared for jobs in industry. In addition, ETH Zürich and industry in Basel must continue to provide, at least to some extent, unrestricted funding and support of the research groups in Zürich to ensure continuing success in the international competition. This will contribute substantially to the survival of a great tradition.
\end{abstract}

Keywords: Chemical industry in Basel · ETH Zürich chemistry · History · Relationship ETH - Basel

*Korrespondenz: Prof. Dr. D. Seebach Laboratorium für Organische Chemie ETH Hönggerberg, $\mathrm{HCl} \mathrm{H} 331$

Wolfgang-Pauli-Str. 10

$\mathrm{CH}-8093$ Zürich

Tel.: +4144632 2990

Fax: +41446321144

E-Mail: seebach@org.chem.ethz.ch
Es ist mir die Aufgabe zugefallen, im Rahmen unseres heutigen Symposiums den einleitenden Vortrag zu halten. Meinem Emeritus-Status entsprechend wählte ich das Thema „ETH Zürich und die Basler Chemie - eine grosse Tradition."

Tradition kommt vom Latein und bedeutet Übergabe, die „Übernahme und Weitergabe von Sitte, Brauch, Konvention, Lebenserfahrung und Institutionen; sie ist das, was die Generationen verbindet und zwischen Vergangenheit und Zukunft Kontinuität stiftet." In der Schweiz ist man - mehr als anderswo - nach wie vor stolz darauf, Gutes zu bewahren und weiterzugeben sowie gleichzeitig besseres Neues zu übernehmen.

So wollen wir bei den Feiern zum 150jährigen Bestehen der ETH Zürich, die einige von uns beinahe liebevoll noch immer „unsere Schule“ oder „das Poly“ nennen, nicht primär die grossen Erfolge in der Ver- gangenheit rühmen, auf die wir stolz zurückblicken, sondern zeigen, was wir heute können, was wir planen und was wir uns zutrauen, in der Zukunft zu bewerkstelligen (Abb. 1).

Wie steht es heute mit der Tradition und dem Verhältnis zwischen der Chemie an der ETH Zürich - nur von ihr soll hier die Rede sein, nicht von anderen Fachbereichen - und der „Basler Chemie,“ d.h. der chemischen Industrie in Basel? Zur Beantwortung dieser Frage ein Blick in die Vergangenheit.

Die Chemie des Poly sollte 1855 ursprünglich wie alle anderen Fächer nur der Lehre dienen. Der erste Chemie-Professor, Städeler, war gleichzeitig an der Universität Zürich tätig, wo zunächst auch die Vorlesungen und Praktika für die Studenten des Poly durchgeführt wurden. Erst 1864 zog man in den Semper-Bau ein (Abb. 2), und als es dort zu eng wurde, baute man ein Chemiegebäude (Abb. 3) direkt dahinter, 


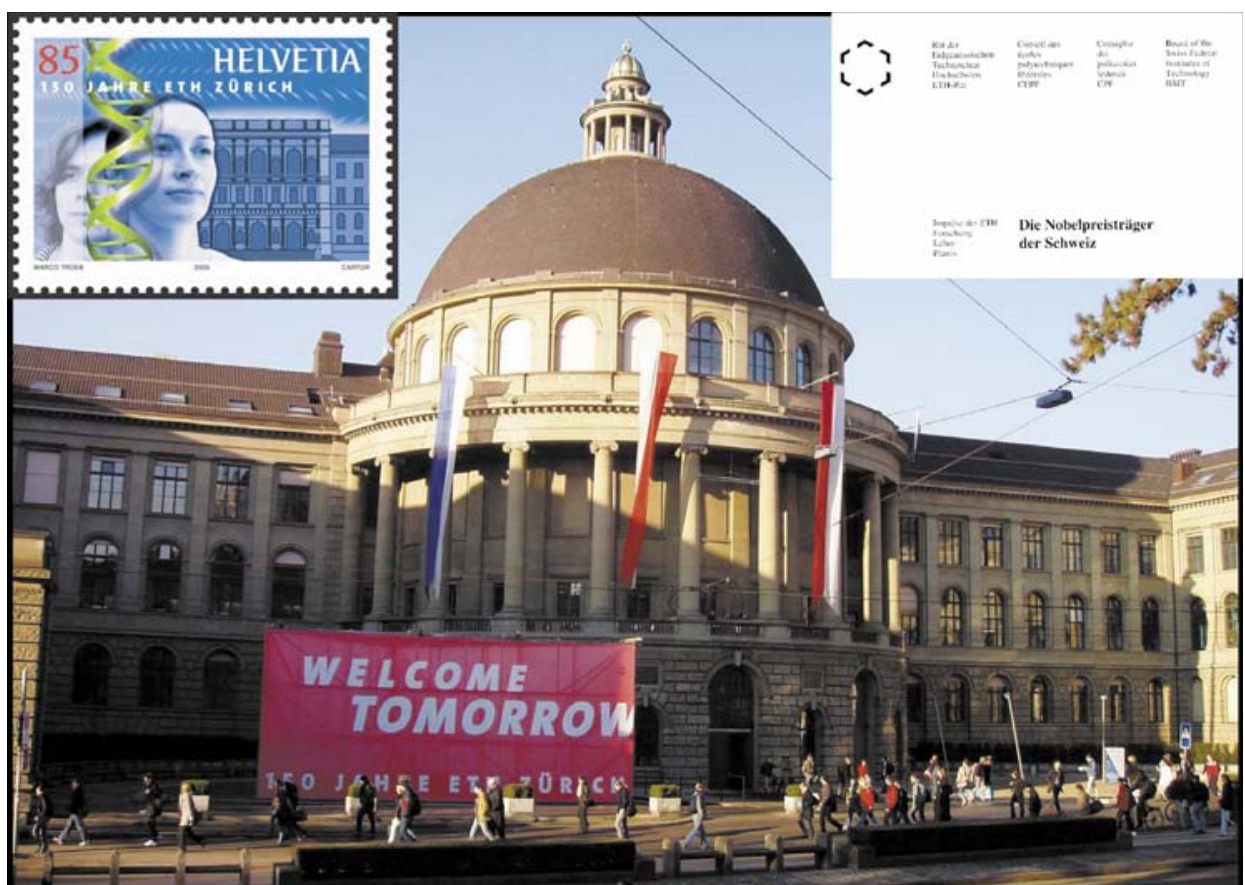

Abb. 1. Das ETH-Hauptgebäude heute. Hier sind die Schulleitung, die Verwaltung und die Hauptbibliothek untergebracht, und es gibt ausser dem Auditorium Maximum zahlreiche grössere und kleinere Hörsäle und Sitzungszimmer. (Quelle: Corporate Communications, ETHZ)
Richtung Zürichberg. Als dieses der Studentenzahl nicht mehr gewachsen war, entstand sukzessive der heute noch bestehende Gebäudekomplex mit Technischer, Anorganischer, Organischer, Physikalischer Chemie und Biochemie (Abb. 4 und 5). Heute sind wir auf dem Hönggerberg in einem sehenswerten Bau - Kosten für den Bund fast 1 Milliarde $\mathrm{CHF}$ - untergebracht, mit den, wie ich meine, besten Chemielabors der Welt, unter einem Dach mit dem Institut für Pharmazeutische Wissenschaften, einem Teil der Biologie und dem Departement für Materialwissenschaften (Abb. 6).

Nicht minder bewegt ist die Geschichte der Chemie-Professoren - hier eine Art Stammbaum (Abb. 7). In allen Bereichen gibt es berühmte Vertreter ihres Faches, z.B. auch G. Schwarzenbach, den Entdecker der Komplexone, in der Anorganischen Chemie, vor allem aber in dem ursprünglich Analytische und Allgemeine Chemie und später Laboratorium für Organische Chemie genannten Bereich (Abb. 8). Bemerkenswert ist, dass von Städeler, der als Gründungsprofessor für Chemie beru-

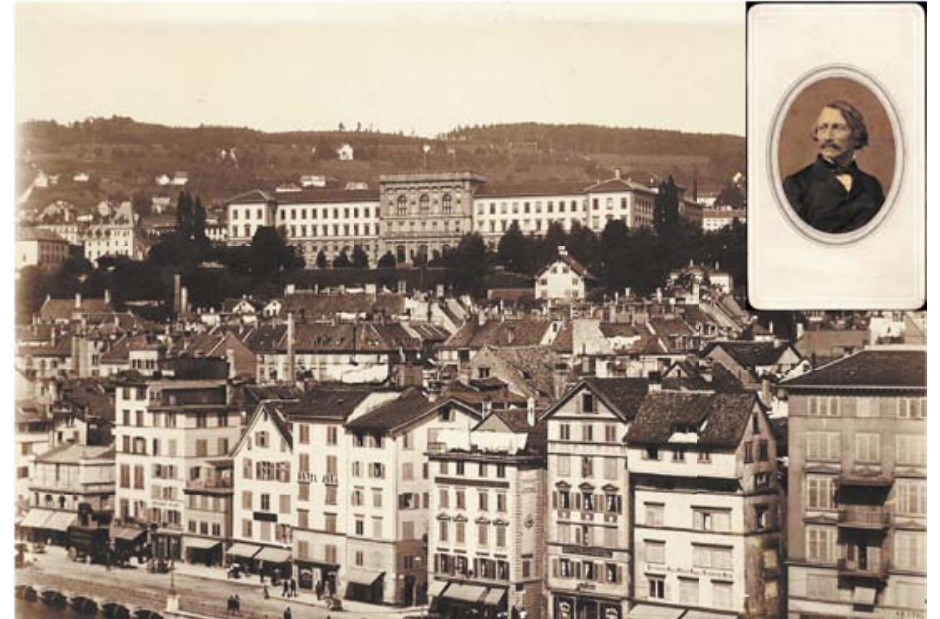

Abb. 2. Das Hauptgebäude des Polytechnikums von Semper - noch ohne Kuppelbau - von der Limmat aus gesehen (um 1900). (Quelle: Bildarchiv ETH-Bibliothek, Zürich)

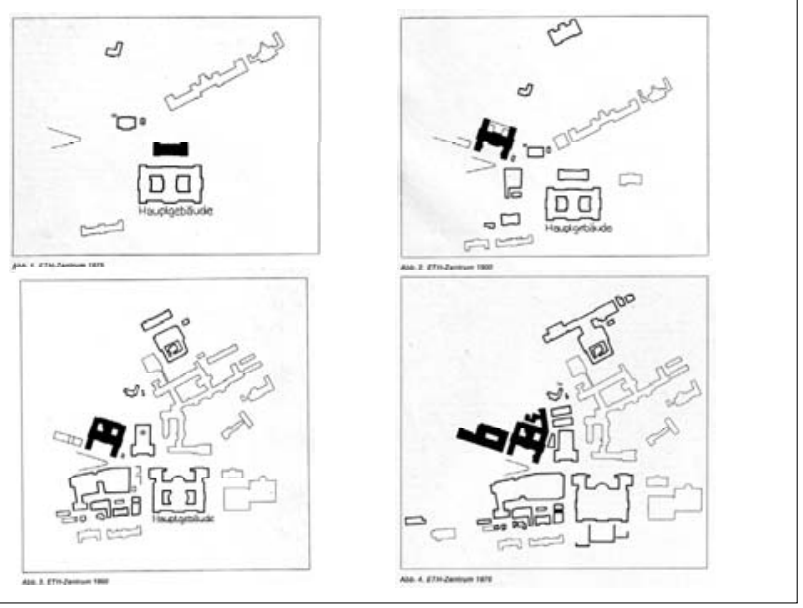

Abb. 4. Skizzen des ETH-Zentrums 1875, 1900, 1950 und 1976 mit den Chemiegebäuden jeweils schwarz hervorgehoben. (Quelle: Die Chemieabteilung der ETH Zürich, Chemische Rundschau 1973)

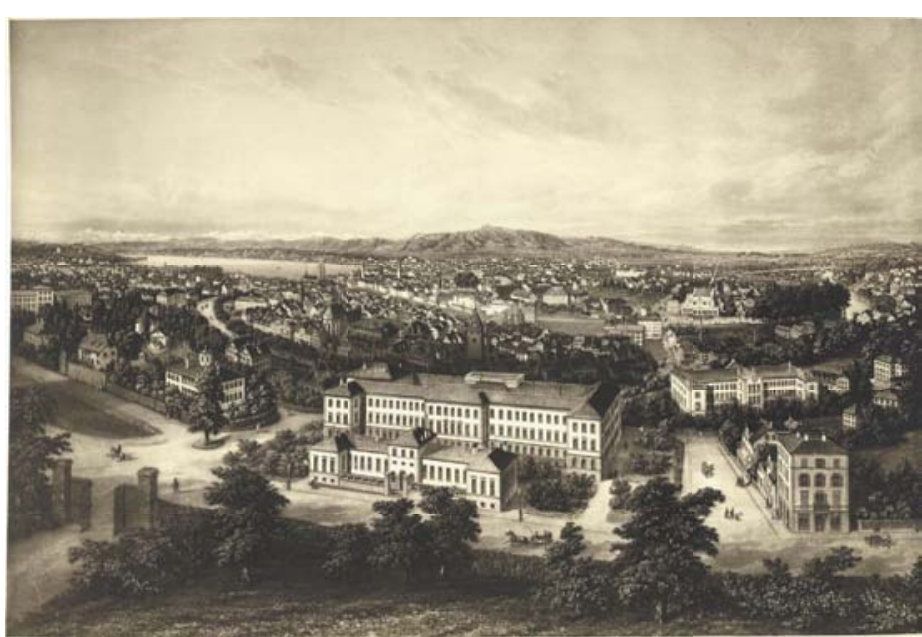

Abb. 3. Der Semper-Bau mit dem ersten Chemie-Gebäude davor - aus Richtung Zürichberg gesehen (1875). (Quelle: Bildarchiv ETH-Bibliothek, Zürich)

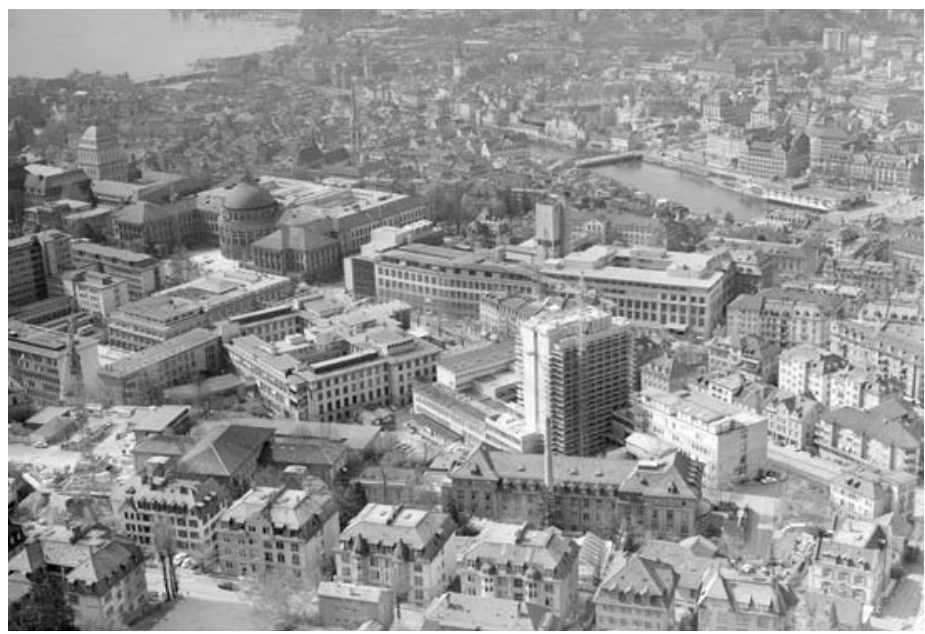

Abb. 5. ETH-Zentrum aus der Luft (1972) mit dem Rohbau des BiochemieHochhauses. (Quelle: Bildarchiv ETH-Bibliothek, Zürich) 


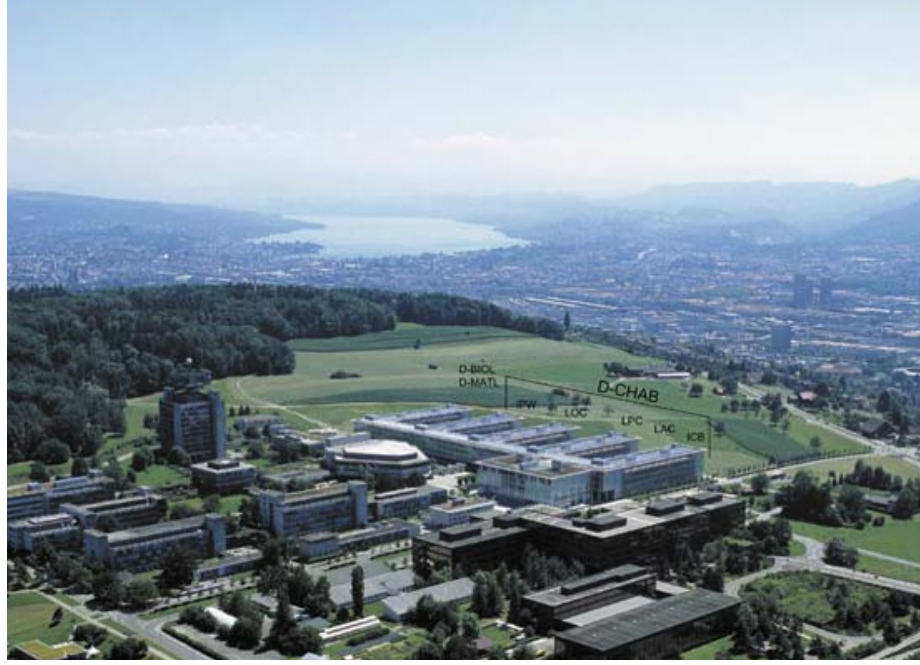

Abb. 6. Chemiegebäude ( $\mathrm{HCl})$ auf dem Hönggerberg (2004) mit Hörsaal-Bibliotheks-Trakt, Zentralgebäude (vor allem für die Lehre) und den fünf „Fingern“, in denen neben einem Teil der Biologie und den Materialwissenschaften die fünf Institute des Departements für Chemie und Angewandte Biowissenschaften (D-CHAB) untergebracht sind. (Quelle: Corporate Communications, ETHZ) fen wurde, nachdem - wohlgemerkt - Kekulé abgesagt hatte, über Wislicenus, Victor Meyer, Hantzsch, Bamberger, Willstätter, Staudinger, Richard Kuhn, Ruzicka bis hin zu Prelog kein einziger geborener Schweizer war. Erster Schweizer in dieser Ahnengalerie ist Albert Eschenmoser. Weiterhin ist - vor Ruzicka - keiner dieser Herren der ETH treu geblieben; sie benutzten das Polytechnikum bzw. die Eidgenössische Technische Hochschule als Sprungbrett vor allem in die - damals - forschungsintensiveren Institutionen Deutschlands. Die Tradition, die besten Fachvertreter weltweit für uns zu gewinnen, blieb erhalten. Heute sind von den 34 Ordinarien in unserem Department nur zwölf in der Schweiz geboren, in der Organischen Chemie sind es zwei von sieben!

Nun zu den Anfängen der Basler Chemischen Industrie, kurz der „Basler Chemie“" oder, wie man in der Region sagt, der „Chemischen“.

Zunächst eine allgemeine Bemerkung: Nur in der Chemie, und oft zu unserem Nachteil, ist der Name zur Bezeichnung der Wissenschaft und deren industrieller Anwendungen gleich - kein Mensch nennt etwa die Firma Siemens, ,physikalische Industrie". Dies rührt wohl daher, dass alle grundlegenden Erkenntnisse in der Chemie enger mit Anwendungen verquickt waren als in den anderen Naturwissenschaften: Justus von Liebig stammte aus einer Drogerie in Darmstadt und seine Analysen organischer und anorganischer Verbindungen wurden Grundlage der Mineraldüngung, und Louis Pasteur aus Dole machte eine bahnbrechende Entdeckung bei der Beschäftigung mit Problemen der französischen Getränkeindustrie.

Auf ähnliche Weise erfolgte die Geburt der eigentlichen chemischen Industrie, das war die Produktion von Farbstoffen. William Perkin, ein Schüler von A.W. von Hofmann am Royal College of London, hatte als 18-jähriger 1856 den ersten Anilinfarbstoff Mauvein im Laboratorium auf

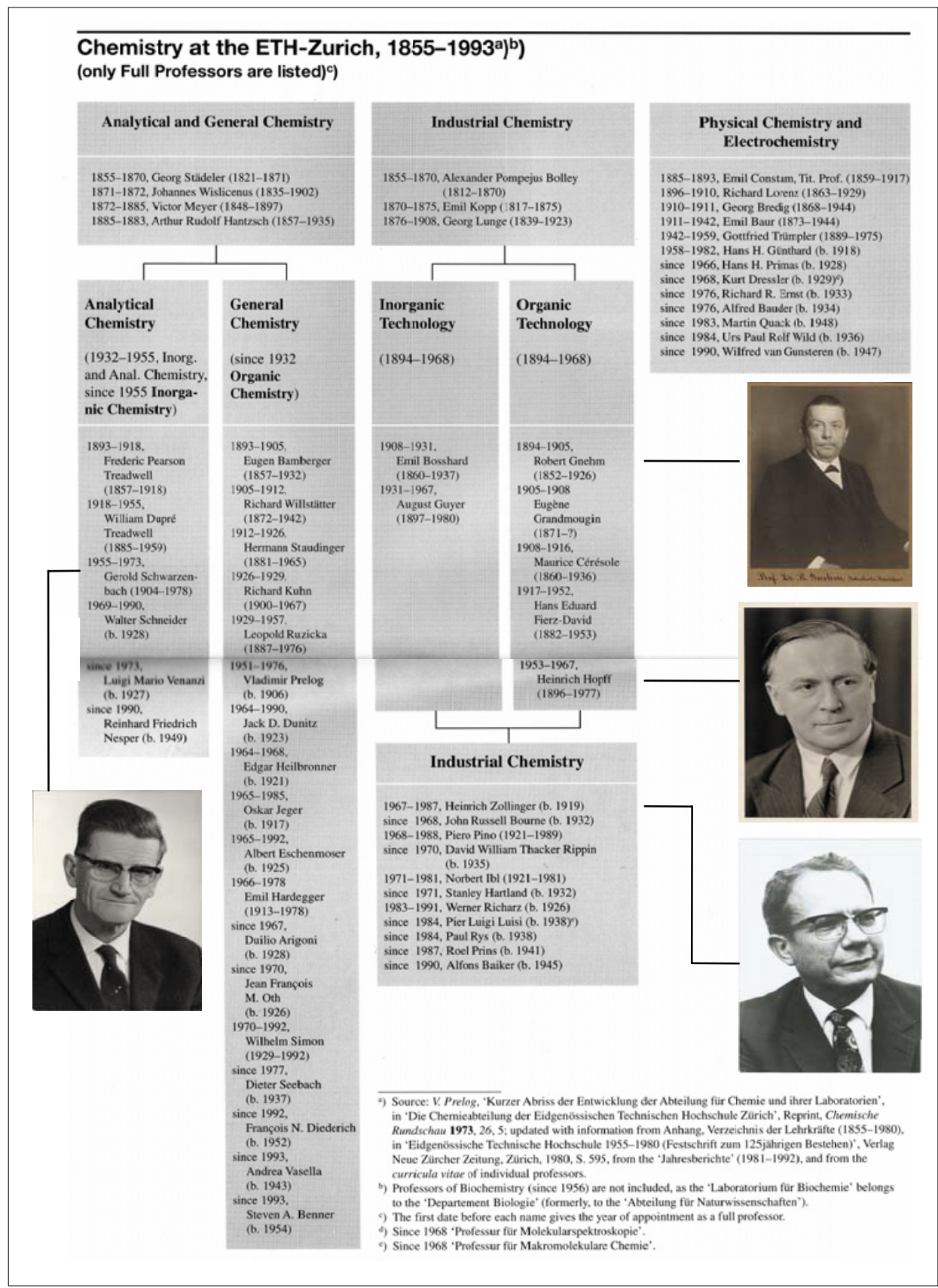

Abb. 7. „Stammbäume“ der Professoren (Ordinarien) in den verschiedenen Chemielaboratorien der ETH Zürich (Stand 1993). (Quellen: Chemistry at the ETH-Zurich, VHCA 1994 und Bildarchiv ETHBibliothek, Zürich) 
dem Speicher seines Wohnsitzes am East End isoliert, eigentlich wollte er Chinin machen! Gegen den Ratschlag seines Lehrers baute er eine Fabrik zur Produktion von Mauvein, zunächst ein Riesenerfolg (Abb. 9). Die damalige Modewelt, bis hin zu den Königshäusern, war verrückt nach dieser Farbe. Mit 22 Jahren Fabrikbesitzer musste Perkin 14 Jahre später verkaufen. Es gab Umwelt- und Gesundheitsprobleme unter den Arbeitern und Anwohnern, aber vor allem hatte ihn die Entwicklung überrollt: England hatte schlechten Patentschutz, Deutsche, Franzosen und Schweizer übernahmen mit grossem Aufwand die Führung. In England spielte die Farbenindustrie schnell eine untergeordnete Rolle, die Textilindustrie ging in der Folge zugrunde, ebenso wie zahlreiche Handelshäuser und Schifffahrtsunternehmen, die z.B. vom Indigo-Transport nach Europa gelebt hatten. Das erinnert alles sehr an die heutige Zeit. Die Chemie ist jetzt dabei, sich - in umgekehrte Richtung - nach Asien zu verlagern; das Buch von Garfield ist hochaktueller, spannender Lesestoff. Perkin war ein Visionär, der auch die chemische Produktion von Lebensmittelzusätzen, Riechstoffen und Medikamenten vorhergesehen hatte!

Während Roche ursprünglich eine Apotheke war, steht am Anfang von Geigy, CIBA und Sandoz, also letztlich von Novartis, die Erfindung von Perkin, die Farbstoffproduktion am Rheinknie, mit dem Standortvorteil, dass die Eidgenossenschaft damals keinen Patentschutz kannte. Pestizide und Pharmaka kamen erst später hinzu. Die Liste der Erfolge ist ebenso imposant wie die vorher gezeigte Ahnengalerie der ETH Zürich, hier nur einige Beispiele (Abb. 10).

Nun zur Partnerschaft in der Forschung zwischen der Chemie der ETH Zürich und der Basler Chemie. Wie erwähnt war das Poly ursprünglich eine reine Schule, und es war ein Chemiker aus der CIBA, Robert Gnehm, der Anfang des letzten Jahrhunderts Präsident des Poly wurde, und der aus seinen Erfahrungen mit der Deutschen Konkurrenz wusste, dass die Industrie in der Forschung ausgebildete Chemiker brauchte. Unter Gnehms Ägide erhielt das Poly 1908 das Promotionsrecht und wurde 1911 in ,Eidgenössische Technische Hochschule " umbenannt. Auch neuerdings wurden Präsidenten wie Jakob Nüesch, Rektoren wie Heinrich Zollinger und Professoren wie Robert Schwyzer, der Gründer des Instituts für Molekularbiologie (Abb. 11), aus der Basler Industrie an die ETH Zürich berufen.

Der Durchbruch zu einer Spitzenposition der ETH Zürich in der Forschung erfolgte in den 20er, 30er und 40er Jahren des letzten Jahrhunderts. Durch umfangreiche Stiftungen - heute würde man vielleicht sagen „Alumni-Beiträge“ - war das Poly
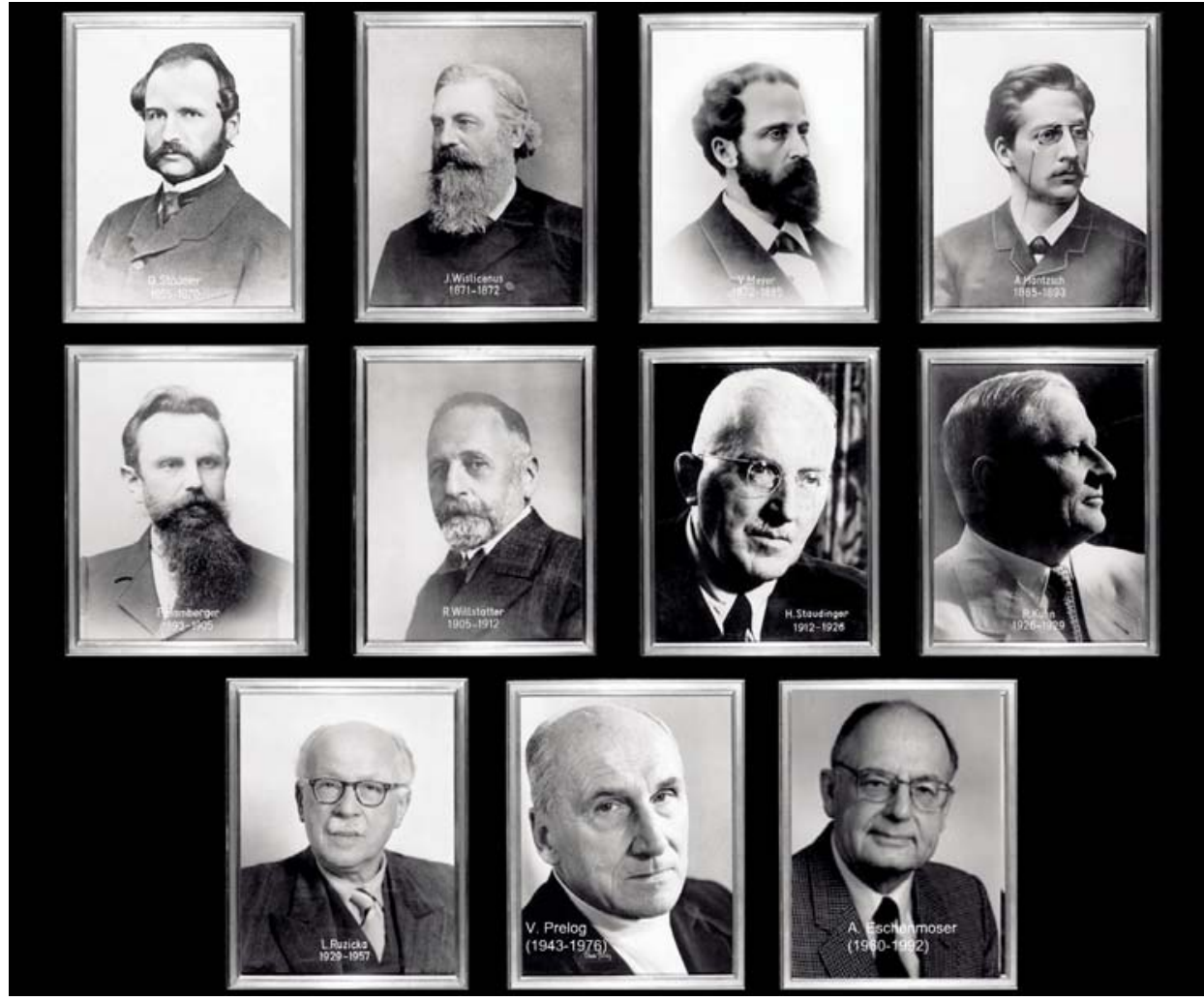

Abb. 8. Die „Ahnengalerie“ des Laboratoriums für Organische Chemie der ETH Zürich. Professoren, die ursprünglich im Bereich Analytische und Allgemeine Chemie, dann Allgemeine Chemie und seit 1932 Organische Chemie tätig waren, mit A. Eschenmoser als erstem geborenen Schweizer. (Copyright: R. Häfliger)
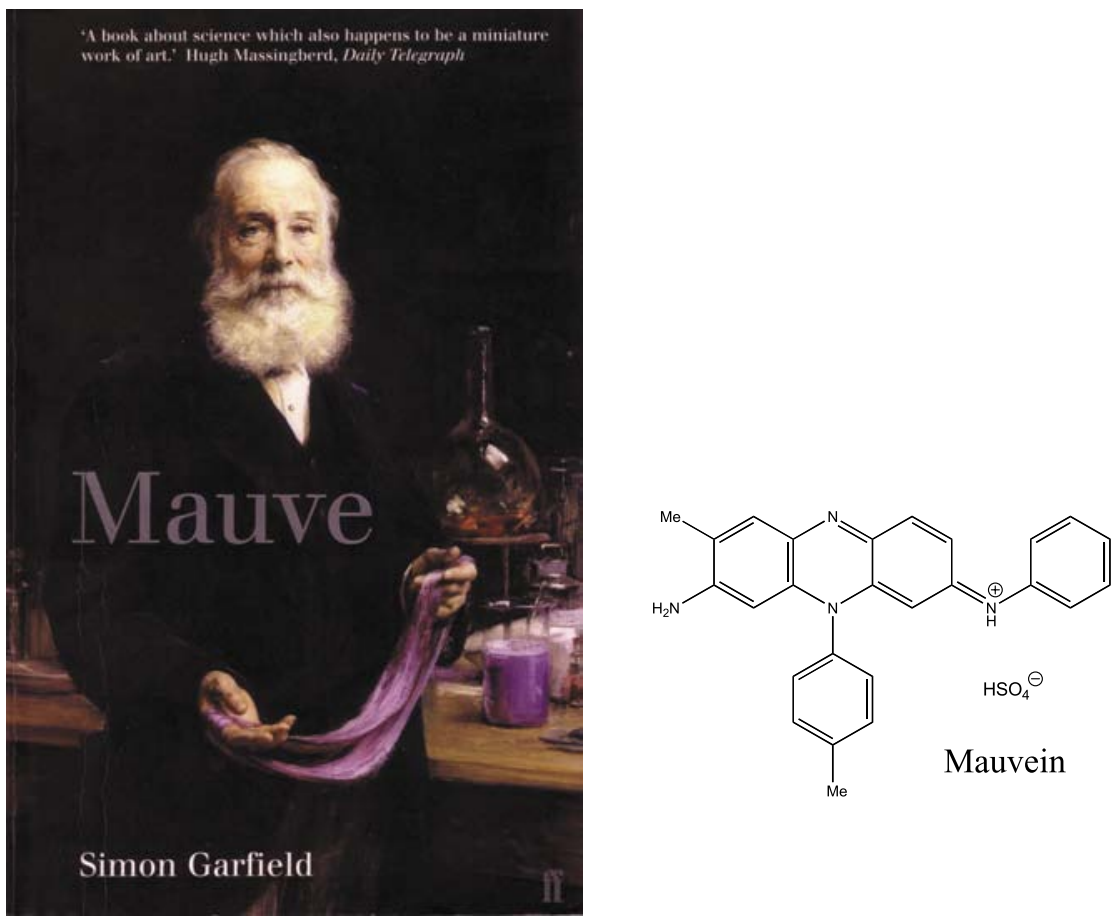

William Henry Perkin (1838-1907)

Abb. 9. Einbanddeckel der Biographie von S. Garfield über W.H. Perkin, Begründer der Chemischen Industrie und Erfinder des Anilinfarbstoffs Mauvein. 'Mauve, How One Man Invented a Colour that Changed the World', Faber and Faber, London 2000.

finanziell so gut ausgestattet, dass es Koryphäen wie Wolfgang Pauli und Paul Scherrer oder Leopold Ruzicka halten konnte. In etwa demselben Zeitraum wuchsen die
Pharmadivisionen der Basler Firmen und die Vitaminsparte, sowie die Riechstoffgruppe der Roche zu weltweit führenden Unternehmen heran. Ohne die Beiträge 


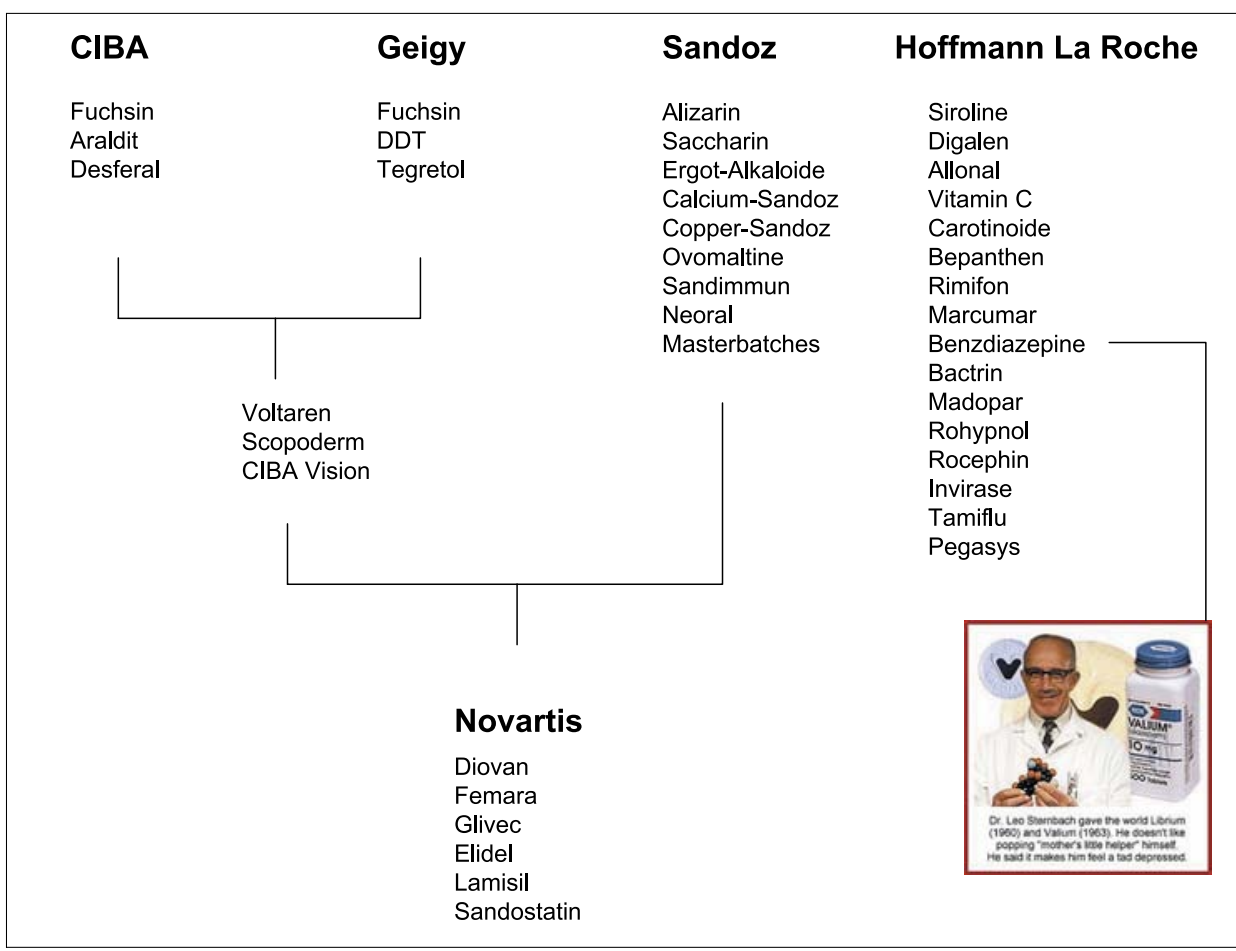

Abb. 10. Die „klassischen“ Basler Chemiefirmen und die Namen einiger ihrer erfolgreichen Produkte. Heute gibt es nur noch die beiden Grosskonzerne Novartis und F. Hoffmann-La Roche.

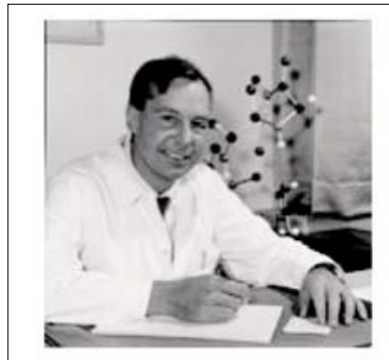

R. Schwyzer (1963-1987)

\section{Gründung Institut für Molekularbiologie}

1971 (1919-1959)

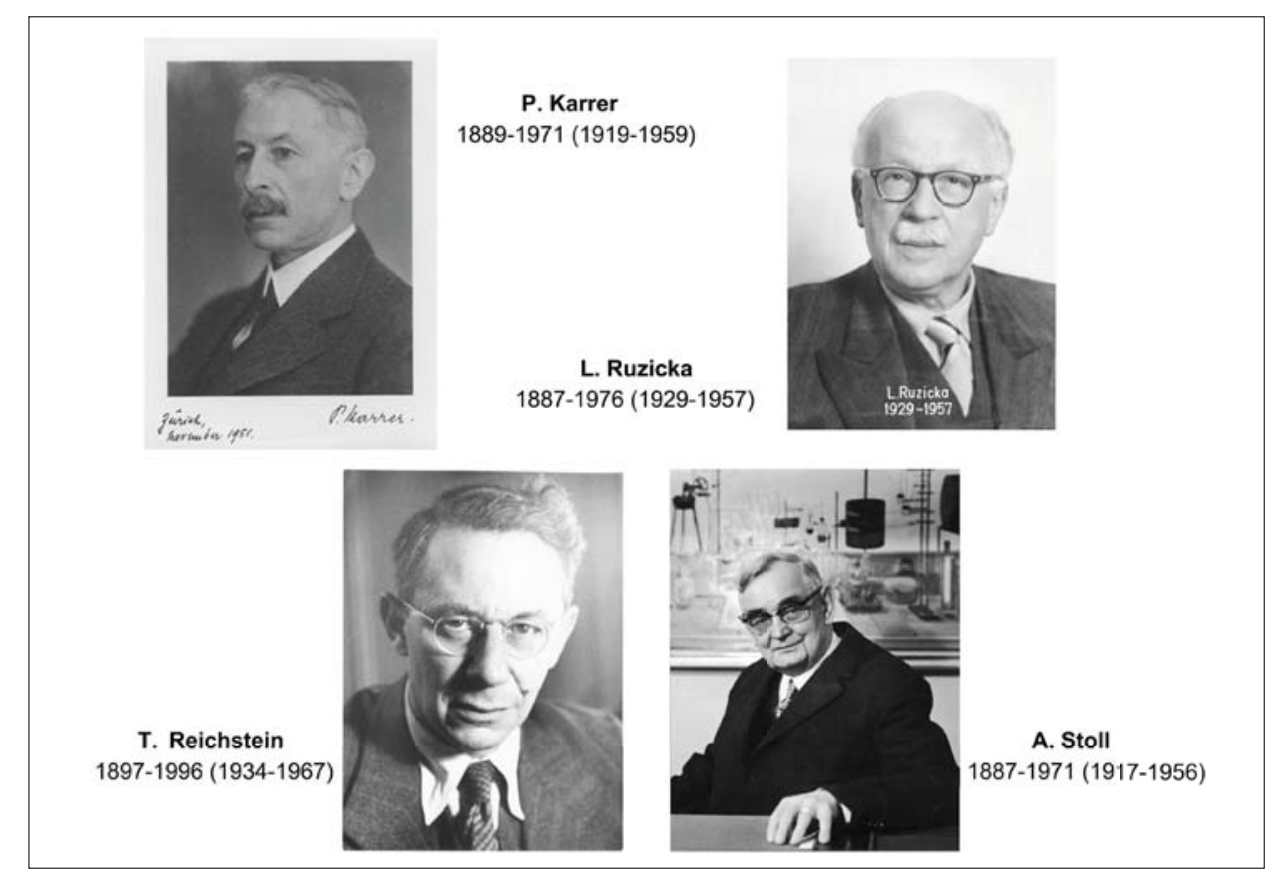

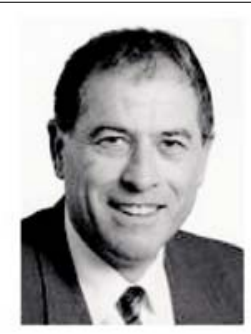

K. Wüthrich (1972-)
Abb. 11. Der Gründer des Instituts für Molekularbiologie und dessen prominentester, kurz vor dem Rücktritt stehender Professor. (Quelle: Bildarchiv ETH-Bibliothek, Zürich)

Abb. 12. Die vier grossen Persönlichkeiten, welche die akademische und industrielle Chemie der Schweiz in den 30er, 40er und 50er Jahren massgeblich prägten. (Quelle: Bildarchiv ETH-Bibliothek, Zürich) von unternehmerisch weitsichtigen und sich für die Region Basel und die Schweiz verantwortlich fühlenden Geschäftsleitungsmitgliedern und Direktoren dieser Firmen schmälern zu wollen, wage ich zu behaupten, dass diese Entwicklung einer einmaligen Konstellation, einer Art Sternstunde zu verdanken war, dem Zusammentreffen der vier Männer Paul Karrer, Tadeus Reichstein, Leopold Ruzicka und Arthur Stoll (Abb. 12), in einer Phase der Chemie, wo die Naturstoffe das Zentrum der molekularen Wissenschaften waren, vor dem Sturmlauf der Biologie und vor Ankunft der Instrumentalanalyse, als Schweizer Uhrmacher- und Tüftler-Qualitäten bei der Laborarbeit entscheidend waren, und als infolge der Wirren vor, während und nach dem Zweiten Weltkrieg hoch begabte Leute, Flüchtlinge und Immigranten, in den sicheren Hafen Schweiz kamen, was schon damals zu einer vorteilhaften, internationalen Atmosphäre in den Basler Firmen führte! Andererseits operierte man in Ermangelung eines nennenswerten Binnenmarktes schon immer viel globaler als z.B. in der Deutschen Chemischen Industrie (während des zweiten Weltkriegs wurde die Firmenzentrale der Roche gar vorübergehend nach Nutley, New Jersey, verlegt!).

Alle vier genannten Herren waren $\mathrm{Na}$ turstoffchemiker, starke Persönlichkeiten, Machtmenschen, eng mit der Basler Chemie verbunden. Karrer forschte an der Uni Zürich über Vitamine und Terpene und war mit der Roche liiert. Ruzicka, ein Schüler Staudingers, führte an der ETH Zürich nach dem Krieg die grösste organisch-chemische Arbeitsgruppe der Welt, die sich mit Steroid-Hormonen, Terpenen und Riechstoffen beschäftigte (Stichwort „Isoprenregel“), eng verbunden mit CIBA und Firmenich. Reichstein, Schüler Ruzickas, hatte sich an der ETH Zürich habilitiert, wurde Professor in Basel, war zunächst Zucker-Chemiker, welcher der Roche die Vitamin-C-Synthese bescherte, später Steroid-Forscher. Stoll, der mit Willstätter von der ETH nach München gegangen war und dann zur Sandoz stiess, zeichnete verantwortlich für deren Aktivitäten auf dem Gebiet der Alkaloide. Die enge Beziehung zwischen den Firmen in Basel und den Professoren, welche ihre Forschungsresultate zur Patentierung anboten, als Konsulenten fungierten und in Verwaltungsräten tätig waren, manifestierte sich in grosszügiger ungebundener oder auch zielgerichteter Finanzierung bzw. persönlicher Vergütung. So konnte Ruzicka durch zwei Besuche in Basel Millionensummen für den Neubau des Biochemie-Hochhauses in der Universitätstrasse beschaffen; er hatte frühzeitig die Bedeutung der Biowissenschaften erkannt und sich an der ETH für die Gründung eines Instituts für Biochemie eingesetzt. Erster Professor wurde Martius (Abb. 13). 


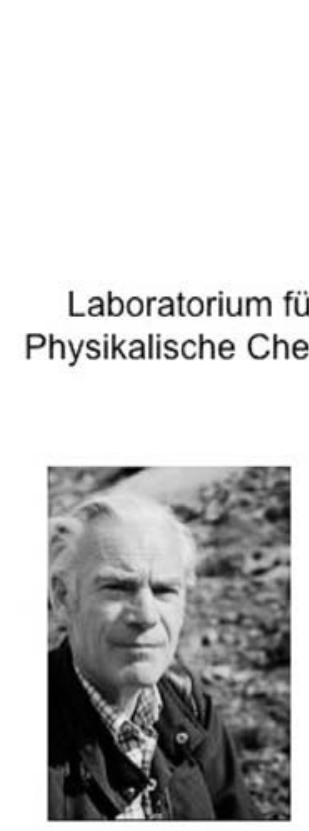

H. H. Günthardt (1952-1982)
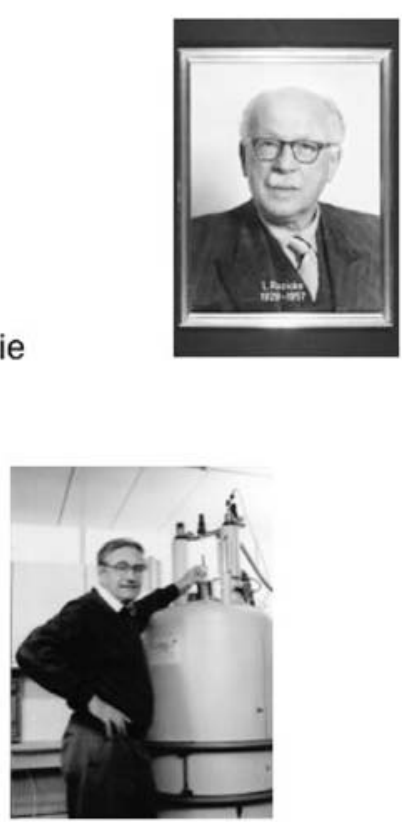

R. Ernst

(1970-1998)

\section{Gründung Institut für} Biochemie

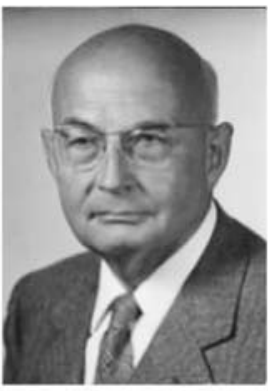

K. Martius

(1956-1976) Zitronensäurezyklus

Abb. 13. L. Ruzicka, der die Entwicklung des Laboratoriums für Physikalische Chemie in Richtung auf instrumentalanalytische Methoden beeinflusste und der für die Gründung des Instituts für Biochemie verantwortlich war. (Quelle: Bildarchiv ETH-Bibliothek, Zürich)

\section{Gerhard Oberkofler \\ Leopold Ruzicka}

\section{Schweizer Chemiker und Humanist}

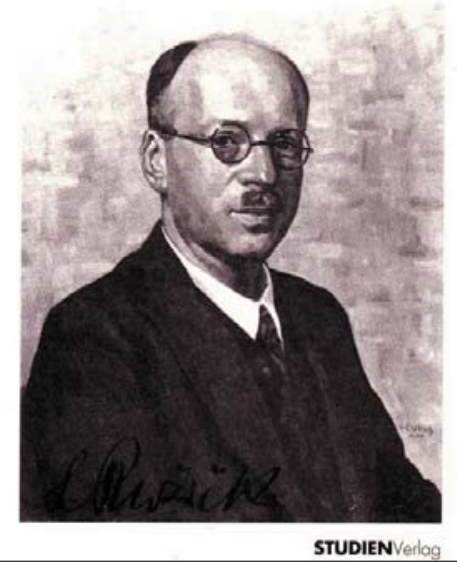
aus Altösterreich

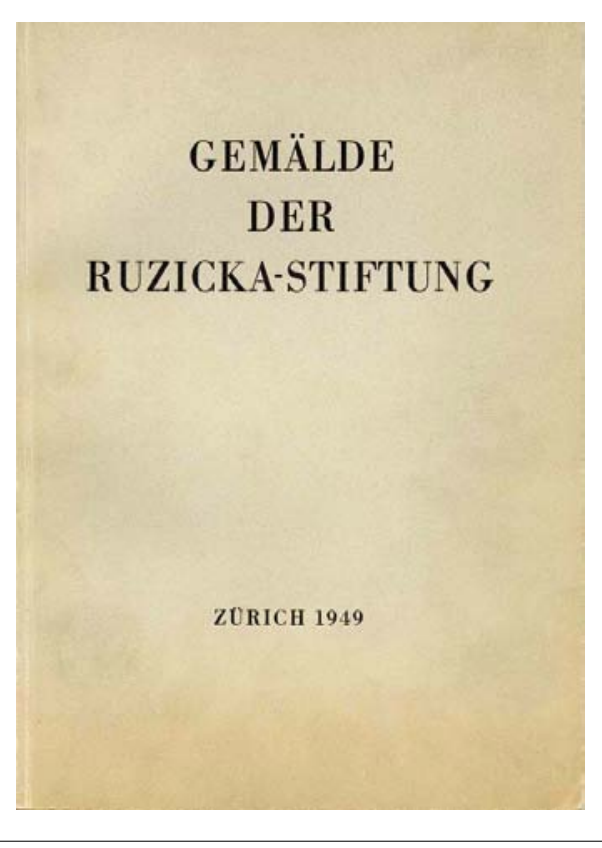

Abb. 14. Leopold Ruzicka, dessen Sammlung holländischer Gemälde noch heute im Kunsthaus Zürich zu bewundern ist.

Visionär sah Ruzicka auch die durch die Instrumentalanalyse zu erwartende Revolution voraus, sein Schüler Günthardt wurde Gründer des Laboratoriums für Physikalische Chemie, wie wir es heute kennen, und siehe, was daraus geworden ist (Abb. 13)! Als die CIBA Ruzicka in den 40er Jahren seinen Anteil aus Einnahmen in den USA für Steroid-Patente überwies - durch oxidativen
Abbau von Cholesterin wurden Steroid-Hormone produziert - meldete er sich im Laboratorium für einige Zeit $\mathrm{ab}$, übergab die Forschung Oskar Jeger, begab sich auf Reisen und legte eine Sammlung flämisch-holländischer Gemälde aus dem 17ten Jahrhundert an (Abb. 14), die er später dem Kunsthaus Zürich vermachte - sie soll heute über 100 Millionen Franken wert sein. Nach einer der zahlreichen Anekdoten-Geschichten Prelogs zeigte ihm Ruzicka - beide stammten aus Kroatien und verfügten über balkanischen Humor und Schlitzohrigkeit - einmal ein Gemälde und fragte ihn, ob es ihm gefalle und ob er es kaufen solle. Prelog antwortete „Ja, Herr Professor, da kenne ich mich doch gar nicht aus, das kann ich nicht beurteilen“, worauf Ruzicka meinte „Ich wollte ja nur einmal die Meinung des kleinen Mannes auf der Strasse hören!“‘

Zurück zum Thema: Ich glaube, man darf wohl sagen, dass der Erfolg der Basler Firmen, vor allem deren Pharmadivisionen, seinen Ursprung an der ETH hatte! Bis in die 70er Jahre versorgte hauptsächlich die ETH-Chemieabteilung die Basler Firmen mit Chemikern. Noch 1977, als ich an die ETH Zürich berufen wurde, waren mehr als die Hälfte aller Chemiker, die ich in Basel traf, ehemalige ETH-ler; sie erwiesen sich durch ihre Ausbildung auf dem Gebiet komplexer Naturstoffe (Strukturaufklärung, Synthese, Stereochemie) den meisten Kollegen in anderen Ländern für Arbeiten zur Erforschung neuer Wirkstoffe überlegen. Die ETH-Professoren waren also nicht nur selbst wichtige Partner der Basler Chemie, sondern vor allem auch die Ausbilder von wissenschaftlichem Nachwuchs für die Firmen. Durch den hohen Bedarf an promovierten Chemikern entstanden starke Arbeitsgruppen an der ETH Zürich, die eine weltweit führende Stellung in der ChemieGrundlagen-Forschung erklommen haben, weil es die Basler Chemie gab! Neben der Chemie hatte auch die ETH-Mikrobiologie wichtige Beziehungen zur Pharmaindustrie in Basel (Abb. 15), während interessanterweise die meisten Biochemiker und Biologen von anderen Schweizer Hochschulen und aus dem Ausland (Deutschland, England) angeheuert wurden.

Seit der erwähnten Sternstunde sind gewaltige Veränderungen in der Chemie der Schweiz und der ganzen Welt eingetreten. Zunächst zur Situation in der Industrie: Nach Fusionen, Fokussierung, Globalisierung und „spin-offs“ sieht das Bild der „Chemischen“ in der Region Basel radikal anders aus. Es erfolgte die Aufteilung nach Pharmazeutischen Wirkstoffen, Riechstoffen und Ernährung, Agrochemie, Feinchemie und Vitaminen, Materialien und Kunststoffen sowie Basischemikalien (Abb. 16). In der Pharma-Forschung hat die Biologie zeitweise die Führung übernommen. Kaum ein Chemiker ist heute Leiter eines Therapie-Bereiches. Die Erfolgsträger auf dem Markt bei Hoffmann-La Roche stammen zur Zeit kaum aus den Chemielaboratorien, sondern sind Produkte der Biotechnologie. Die Instrumentalanalyse spielt eine zentrale Rolle, vor allem Röntgenstruktur, Massenspektrometrie und NMR-Spektroskopie. Die grösste Revolution hat aber wohl die Informationstechnologie bewirkt - Stich- 


\section{Mikrobiologie der ETHZ}

\section{E. Gäumann}

\section{H. Zähner}

R. Hütter

\section{W. Keller-Schierlein}

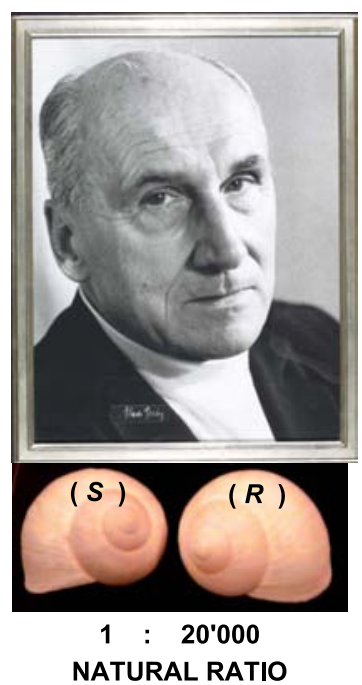

\section{Narbomycin} Sidermycine Rifamycin Nonactin Boromycin Antibiotica

Abb. 15. Vladimir Prelog, der wie Ruzicka kroatischen Ursprungs war und aus Sarajewo stammte, hat durch seine Arbeiten auf dem Gebiet der Strukturaufkärung von mikrobiellen Stoffwechselprodukten und derVerwendung von Mikroorganismen fürenantioselektive Transformationen die Molekularbiologie an der ETH Zürich und das Antibiotika-Geschäft in der Basler Industrie beeinflusst. Den Nobel-Preis erhielt er für grundlegente Arbeiten auf dem Gebiet der Stereochemie, siehe z.B. die Cahn-IngoldPrelog (CIP) Nomenklatur.

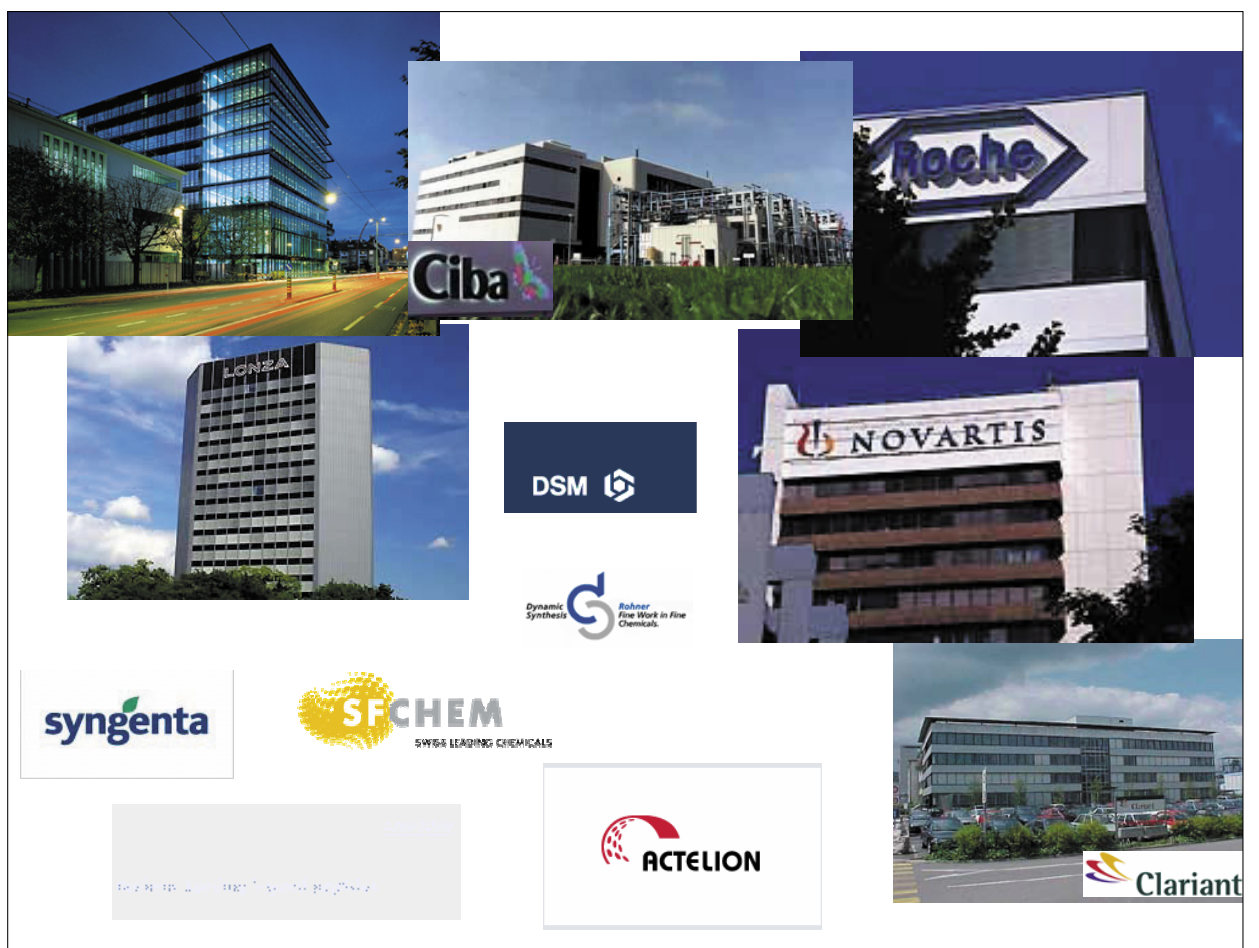

Abb. 16. Das heutige Bild der „spezialisierten Basler Chemie“. Von den Namen der drei Klassiker (Abb. 10) ist nur CIBA geblieben. SANDOZ heisst die Generika-Abteilung von Novartis.

worte: Datenverarbeitung, Modellieren, Chips-Technologie, Robotics, Combichem, High-Throughput-Screening, Substanzbibliotheken (Abb. 17). Chemische Generalisten und Chemieingenieure spielen erst bei der Chemischen Entwicklung, Pilotierung und Produktion eine zentrale Rolle, und hier ist heutzutage eine Verschiebung Richtung Asien zu beobachten. Ein kürzlich erfolgter, besorgter Aufruf unter der Federführung von Jean-Marie Lehn weist auf die Gefahr hin, dass die chemische Forschung an den
Hochschulen mit dieser Verschiebung auch aus Europa zu verschwinden droht; der an die Präsidenten des Europäischen Parlamentes und der Europäischen Kommission gerichtete „offene“ Brief hat den Titel: „Why we should keep chemistry research and chemical plants in Europe!". Die so erfolgreiche Novartis hat ihre Pharma-Forschungszentrale nach Cambridge (USA) verlegt; dort wurden binnen kürzester Zeit hunderte von neuen Stellen geschaffen und mit Forschern und Technikern aus der ganzen Welt, vor allem aber aus den USA, besetzt. Unter den ingesamt 20 Mitgliedern von Verwaltungsrat und Geschäftsleitung der Novartis befindet sich übrigens heute nur ein einziger Chemiker.

Nicht minder dramatisch sind die Veränderungen an den Chemieabteilungen der Hochschulen, auch an der ETH Zürich. Die vorderste Front der molekularen Wissenschaften hat sich von der Naturstoffchemie zur Chemie der Übergangsmetalle, zur supramolekularen Chemie, den Material- und den Biowissenschaften hin verschoben (Abb. 18, oben). Es ist leichter, Forschungsfinanzierung auf diesen Gebieten $\mathrm{zu}$ beschaffen, deren Bearbeitung oft synthetisch nicht sehr anspruchsvoll ist. Dadurch entsteht die Gefahr, dass die Hochschulen nicht mehr genügend allgemein-synthetisch ausgebildete Chemiker für die chemische Entwicklung, Pilotierung und Produktion in der Industrie hervorbringen. Es gibt kaum mehr „reine“ Chemie-Departmente; seit die Chemie an der ETH Zürich als fünftes Laboratorium das Institut für Pharmazeutische Wissenschaften aufgenommen hat, sind wir das Departement für Chemie und Angewandte Biowissenschaften (D-CHAB). In einer Diskussion in Chemical \& Engineering News, der wöchentlichen ACS-Hauszeitschrift, wurde kürzlich der Vorschlag gemacht, die American Chemical Society in Society for Molecular Sciences and Engineering umzubenennen, wobei der Wegfall von „American“ und von „,chemical" ebenso bemerkenswert ist wie die Tatsache, dass damit wohl die Synthese zu einem Ingenieurfach würde (,Molecular Engineering"). Weitere Gedanken zur Zukunft der Chemie werden in den in Abb. 19 angegebenen Artikeln geäußert.

Aber nicht nur der Schwerpunkt der Chemie-Forschung, sondern auch die finanziellen Rahmenbedingungen an den Hochschulen, die von öffentlicher Hand finanziert werden, haben sich verschoben (Abb. 18, unten). Die Forschung wird immer teurer, die Mittel werden knapper, und die ausgebildeten Spitzenkräfte kommen nicht mehr nur der eigenen Ökonomie zugute - also nach dem Motto „Schweizer studieren Chemie, promovieren an der ETH und gehen in die Basler Industrie, welche wiederum Steuern in die Bundeskasse 
zahlt". Stattdessen stammen immer mehr unserer Forscher aus aller Herren Länder und bewerben sich nach dem Abschluss um Stellen auf der ganzen Welt. Dadurch ergibt sich die konkrete Frage: Kann sich der Schweizer Steuerzahler eine globale Kaderschmiede leisten?

Zur Linderung der Finanznot werden weltweit Professoren an Hochschulen, wie Angestellte von Firmen, verpflichtet, ihre Forschungsergebnisse $\mathrm{zu}$ patentieren, und ermuntert, Forschungsaufträge von der Industrie einzuholen - mit Geheimhaltungsverpflichtungen (!) und Abführung von „Overhead"-Zahlungen an die Hochschule. Es können gemeinsam mit der Industrie Projekte formuliert werden, die man an der Hochschule bearbeitet, und die Ergebnisse unterliegen Publikationssperrfristen. Ein „outsourcing“ von Industrieforschung in die billigeren Hochschulen ist in anderen Ländern, wie z.B. Grossbritannien, schon teilweise eingetreten. Ein Hochschullehrer kann nicht mehr offen und öffentlich seine Forschungsergebnisse diskutieren und eine Entdeckung, die er diese Woche macht, in der nächsten Woche bei einem Vortrag an einer anderen Universität oder einem Kongress vorstellen. Die kulturelle Mission, welche die Hochschule auch hat, nämlich das Wissen zu mehren, steht auf dem Spiel, siehe auch den besorgten Aufruf von Papst Johannes II in Abb. 20.

Es ist klar, dass sich in diesem veränderten Umfeld die traditionellen Beziehungen zwischen der ETH Zürich und den Basler Firmen, die jetzt globale Unternehmen sind - die meisten sehen sich selbst schon gar nicht mehr als Chemie-Firmen - auch geändert haben. Basel droht zum Ort der Verwaltungszentralen zu werden und bleibt nur teilweise Pharma-Forschungsstandort, die chemische Entwicklung ist in Gefahr abzuwandern und die Produktion hat das schon weit gehend getan. Man ist aber in Basel immer noch ein wenig stolz auf die ETH Zürich, was aus folgenden Aktivitäten ersichtlich ist: Dem Department für Biologie wurden mehrere Assitenz-Professuren gestiftet; die aus Vertretern der Industrie zusammengesetzte Kontaktgruppe für Forschungsfragen, $\mathrm{KGF}$, entsendet einen Vertreter in alle ETH-Chemie-Berufungskommissionen und finanziert im Rahmen der Berufungsverhandlungen einen Teil der Erstausstattung; gewisse ETH-Einheiten erhalten jährliche Zuwendungen zur Bezahlung von Doktoranden oder zur Finanzierung von Gastvorlesungen und von Auszeichnungen; auch wenn eine intensive gegenseitige Befruchtung der Forschungsaktivitäten wie zu Ruzickas Zeiten kaum mehr vorkommt, haben einige Hochschullehrer einen Konsulentenvertrag mit einer der Firmen. Sie beraten die Kollegen in Basel auf ihrem Spezialgebiet, wofür ihnen Gel-

\section{Industrie}

Fusionieren, Fokussieren, Globalisieren

Pharma
Chemie
Biologie
Biotechnologie
Diagnostik
Instrumentalanalyse
Informationstechnologie
Miniaturisierung
Roboterisierung

Riechstoffe Ernährung

Agro

Feinchemie Vitamine

Materialien

Kunststoffe

Basischemikalien

Verschiebung der Märkte, der Forschungs- und der Produktionsstandorte

$$
\text { Europa } \rightarrow \text { USA } \rightarrow \text { Asien }
$$

Abb. 17. Aufteilung der Basler Chemie nach Einsatzgebieten der Produkte und Verlagerung von Märkten, Forschungszentren, Chemischer Entwicklung und Produktion in die ganze Welt.

\title{
Hochschule
}

\author{
Zentrum der Molekularen Wissenschaften \\ Naturstoffchemie \\ "klassische" Synthese \\ $\checkmark$ Übergangsmetallchemie \\ Katalyse \\ Supramolekulare Chemie \\ Materialien

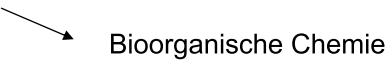 \\ Biologie ("Genomics", "Proteomics") \\ Biomedizin
}

\section{Finanzierung der Forschung}

Staatliche Budgets

Ungebundene Drittmittel von der Industrie

Lehr-, Forschungs-, Publikationsfreiheit

Verpflichtung zur Patentierung der Ergebnisse aus den Universitäten
"Outsourcing" von Forschung Industrie $\rightarrow$ Hochschule
Firmengründungen durch Hochschullehrer

Abb. 18. Weltweite Verschiebung der Forschungsschwerpunkte auf dem Gebiet der Molekularen Wissenschaften an den Hochschulen, wobei die klassischen Grenzen zwischen Anorganischer Chemie, Biochemie, Organischer Chemie, Physikalischer Chemie, Technischer Chemie und Materialwissenschaften ebenso verschwinden wie diejenigen zwischen den „Grundfächern" Mathematik, Physik, Chemie, Biologie, Medizin oder auch den Ingenieurwissenschaften. Gleichzeitig führt der wachsende Finanzbedarf der Hochschulforschung zu deren Kommerzialisierung und Applikationsrelevanz.

der zur Ausrichtung von DoktorandenStipendien zur Verfügung gestellt werden. Dadurch kommen Forschungskontakte zustande, und es ist sicher gestellt, dass Praxisrelevanz von Ergebnissen aus der Hochschule erkannt wird. Umgekehrt können in Diskussionen mit Kollegen in Basel Fragestellungen auftauchen, deren Verfolgung Grundlagenforschung in einem Umfang erfordert, der die Möglichkeiten des Industriechemikers übersteigt, so dass der Hochschullehrer manchmal 


\section{American Chemical Society \\ Society for Molecular Sciences \& Engineering Molecular Sciences - Wissenschaft \\ Chemical \& Engineering News, Nov. 8, 2004}

Organische Synthese - wohin?

D. Seebach, Angew. Chem. 102, 1363 - 1409 (1990)

\section{Chemie: auf zu neuen Zielen - Gedanken zur Zukunft der Chemie \\ G. Whitesides, Angew. Chem. 116, 3716 - 3727 (2004)}

\section{Chemie: ein Schlüssel zur Zukunft}

US-NationalAcademy of Sciences (Nachrichten aus der Chemie 52,1139 - 1143 (2004))
The pre-eminence of the profit motive in conducting scientific research ultimately means that science is deprived of its epistomological character, according to which its primary goal is discovery of the truth.

The risk is that when research takes a utilitarian turn, its speculative dimension, which is the inner dynamic of man's intellectual journey, will be diminished or stifled

The Pope

Abb. 19. Diskussionen und Artikel über die Zukunft der Chemie.

Abb. 20. Sorge des Papstes Johannes Paul II.

Tabelle. Berufsfelder für Abgänger des ETH-Departements für Chemie und Angewandte Biowissenschaften

Pharmaforschung (Agroforschung)

Entdeckung von Wirkstoffen

Synthese kleiner Sustanzmengen

Combichem

Enzymchemie

Biochemie

Medizinalchemie

Molekularbiologie

Biologie

Zellbiologie

Biomedizin

Medizin

(Pflanzenwissenschaften)

\section{chemische}

Wirkstoffsynthese

Pilotierung

Produktion

Biotechnologische Produktion

\section{Materialien}

für die Elektronik

Datenspeicher

Chips

DVD

LCD

\section{Basischemie}

Farbstoffe, Pigmente

Veredlungsstoffe für Metalle, Textil,

Leder, Papier

Additive

Polymere, Kunststoffe, Fasern

Katalysatoren

\author{
Zentrale Technologie \\ Analytik \\ NMR-Spektroskopie \\ Massenspektrometrie \\ Kristallstrukturbestimmung \\ Isolierung/Strukturaufklärung \\ von Naturstoffen \\ Modellieren \\ Substanzbibliotheken \\ High-Throughput Screening \\ Strukturbiologie
}

\section{Entwicklung und Produktion}

pharmakologische

Bioverfügbarkeit

Toxizität

Metabolismus

Galenik

\section{Feinchemie}

Pharma- und Agro-Zulieferer

Vitamine

Riech- und Geschmackstoffe

Pestizide

Auftragsynthese und Lohnproduktion

für Pharma und Agro

\section{Grundstoffe}

Produkte aus dem Cracker

Lösungsmittel

Ammoniak

Chloralkali

Schwefel- und Salpetersäure

Düngemittel mit Anregungen für eine neue Forschungsrichtung nach Zürich zurückkehrt.

Um die „Gunst der Basler“ müssen wir mit anderen Hochschulen konkurrieren: Hochschullehrer aus aller Welt fungieren als Berater in Basel und in den anderen Forschungszentren der Firmen und unsere Abgänger haben keine "Standortvorteile“ mehr.

Was muss das Departement CHAB tun und was hat es bereits getan, damit die traditionsreiche Beziehung zur ,Basler Chemie", wie sie sich heute präsentiert, auch in Zukunft besteht und sich weiter entwickeln kann?

Betrachtet man die Studiengänge des Departements und die Themen der Arbeitsgruppen in den fünf Forschungseinheiten, den Laboratorien für Anorganische, Organische, Physikalische Chemie, und den Instituten für Chemie- und Bio-Ingenieurwissenschaften und für Pharmazeutische Wissenschaften im Akademischen Führer 2004/2005 der ETH und vergleicht sie mit den Berufsfeldern in der Industrie (Tabelle), sollte es für jeden unserer Abgänger geeignete Stellen geben. Dabei kommt den Bewerbern zugute, dass die Lehre und noch vielmehrdie Forschung in unserem Departement heute in hohem Masse interdisziplinär ist, auch über die Departementgrenzen hinaus. Viele Projekte werden zusammen mit Mitgliedern der Departemente Biologie, Materialwissenschaften und Umwelt-Naturwissenschaften bearbeitet, und es gibt fachübergreifende, sogenannte virtuelle Zentren, z.B. für Analytik, Functional Genomics und Nanotechnologie. Dabei spielt die Chemie eine immer wichtigere Rolle. Wir verstehen die in lebenden Organismen ablaufenden komplexen Prozesse immer besser auf molekularer und atomarer Ebene, wir können Art, Aufenthaltsort und Bewegung von Molekülen innerhalb von Zellen bestimmen, wir können Wechselwirkungen 


$$
\begin{aligned}
& \text { La chimie crée son objet. } \\
& \text { Cette faculté créatrice, } \\
& \text { semblable à celle de l'art lui même, } \\
& \text { la distingue essentiellement } \\
& \text { des sciences naturelles et historiques. } \\
& \text { M. Berthelot (1860) }
\end{aligned}
$$

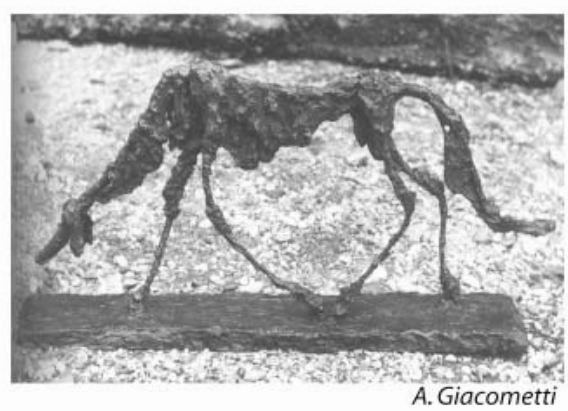

Abb. 21. Definition der Chemie von Berthelot, der die Kreativität des Chemikers mit derjenigen des Kunst-Schaffenden vergleicht.

von niedermolekularen Verbindungen mit aktiven Zentren von Enzymen modellieren und Ligand-Rezeptor-Bindungen nachahmen, und wir können Nanostrukturen gezielt synthetisieren, um nur einige Beispiele zu nennen. Die vorderste Front der molekularen Wissenschaften bewegt sich in Richtung Biologie, Medizin, Materialwissenschaften; es ist der Chemiker, welcher die Reaktivität von Verbindungen im „Gefühl“" hat, welcher in dreidimensionalen Strukturen „,denkt“, welcher scharfe analytische Kriterien anlegt und welcher Bindungen in Molekülen brechen und knüpfen, d.h. neue Verbindungen herstellen kann. Aus meiner Sicht ist das „Herzstück der Chemie“ die Synthese, was schon Berthelot vor fast 150 Jahren zu ihrer Definition herangezogen hatte (Abb. 21). Das gilt auch gerade in den ,,modernen“" Gebieten der Chemie, wo „Ziele der Synthese nicht mehr Moleküle um ihrer selbst willen sind, sondern die Funktionen und Eigenschaften der hergestellten Gebilde“, aber ,auch auf diesem Gebiet werden in Zukunft die interessantesten Ziele und die anspruchsvollsten Themen von denjenigen erfolgreich bearbeitet werden, die synthetisieren können" (Zitat aus meinem in Abb. 19 angegebenene Artikel).

Es ist also wichtig, dass wir im Departement unseren Studenten, Doktoranden und Postdoktoranden ein solides Wissen in den Grundfächern, der Anorganischen, Organischen und Physikalischen Chemie beibringen, bevor sie sich spezialisieren, damit wir nicht Dünnbrettbohrer hervorbringen, die von allem etwas und von nichts alles wissen bzw. verstehen. Gleichzeitig müssen sie wenigstens die Sprache der „Nachbarwissenschaften" erlernen, um in interdisziplinären Teams der Chemie die ihr gebührende führende Rolle zu verschaffen. Dazu haben wir z.B. schon vor Jahren im Curriculum der Studenten mit Hauptfach Chemie in den ersten beiden Semestern eine dreistündige Pflicht-Vorlesung in Biologie eingeführt.

Von Seiten der „Schule“ ist es ebenso wichtig, dass die solide Grundausstattung unserer ETH-Professuren mit Stellen, Sachmitteln, Geräten und Räumlichkeiten erhalten bleibt, die es uns erlaubt, in gewissem Umfang hochriskante, langwierige Forschungsziele in Angriff zu nehmen, für die wir keine Anträge schreiben oder Berichte verfassen müssen, und für die ein Finanzierungsantrag heikel wäre.

In diesem Zusammenhang sollte man auch in der Industrie nicht vergessen, wie wertvoll für unsere Professoren eine ungebundene finanzielle Unterstützung (,,unrestricted funding") ist, um einen zusätzlichen Freiraum für deren Forschung zu schaffen, und dass Abgänger der ETH, welche in einem solchen Freiraum aufgewachsen sind, meines Erachtens „,bessere“ Mitarbeiter in den Firmen werden.

Mit den bestausgebildeten Doktoranden und Postdoktoranden, einer gleich bleibend guten Grunddotation der Professoren und unbürokratischer Grosszügigkeit der Basler Firmen wird es uns auch künftig gelingen, gegen schärfste Konkurrenz anderer Top-Schulen die besten Forscher und Hochschullehrer aus der ganzen Welt an die ETH zu berufen, und wir brauchen uns keine Sorgen um den Fortbestand der traditionell hervorragenden Beziehung zwischen der Chemie an der ETH Zürich und derjenigen am Rheinknie zu machen.

\section{Dank}

Den Kollegen D. Arigoni und A. Eschenmoser danke ich für erleuchtende Diskussionen zum Thema meines Vortrags in Basel. Den Herren A.K. Beck, Dr. M. Limbach, Dr. J. Gardiner und R. Häfliger gilt mein Dank für die Erstellung der Abbildungen. Frau Dr. C. Bärtsch (ETHBibliothek, Spezialsammlungen Bildarchiv), Frau E. Gantner (Novartis-Firmenarchiv) sowie die Herren Dr. E. Zass (Informationszentrum Chemie, Biologie, Pharmazie, ETH Zürich) und C. Becker (Kunsthaus Zürich) haben Bildmaterial für die Abbildungen zur Verfügung gestellt.

Eingegangen am 6. Juli 2005
Wertvolle Quellen bei der Abfassung des Manuskriptes:

- V. Prelog, 'My 132 Semesters of Chemistry Studies, Profiles, Pathways, and Dreams', J.I. Seeman, Series Editor, American Chemical Society, Washington, DC, 1991.

- M.V. Kisakürek, E. Heilbronner (Eds.), 'Highlights of Chemistry as Mirrored in Helvetica Chimica Acta', Verlag HCA, Basel and VCH, Weinheim, 1994. 